\title{
Repurposing COVID-19 Drive-Through Testing Centers for Mass Vaccination
}

This article was published in the following Dove Press journal:

Journal of Multidisciplinary Healthcare

\section{Edward Kim}

Medical School for International Health, Ben Gurion University of the Negev, Beer Sheva, Israel
Correspondence: Edward Kim Ben Gurion University of the Negev, P.O. Box 653, Beer Sheva 841050I, Israel Email contact@eddiewkim.com
Abstract: Throughout the COVID-19 pandemic, drive-through testing centers have proven more effective in expanding testing capacity than traditional clinic models. If this infrastructure is repurposed and expanded for a large-scale vaccination campaign, it can facilitate throughput, reduce resource use, and overcome barriers associated with vaccine adoption and distribution.

Keywords: public health, screening, pandemic, coronavirus, SARS-CoV-2, vaccination

\section{Introduction}

In response to the coronavirus disease 2019 (COVID-19) pandemic, many countries including Israel and South Korea have improved their testing capacity by implementing drive-through testing centers. ${ }^{1,2}$ As countries continue to navigate the ongoing pandemic, they are moving beyond the goals of increasing testing capacity and focusing on vaccine development. ${ }^{3}$ Vaccines are critical to increasing immunity and decreasing morbidity. ${ }^{4}$

While it is imperative to invest the time and resources required for vaccine development, vaccine distribution also necessitates advanced planning and reevaluation of current practices. Even though drive-through testing centers were originally operationalized to expand testing capacity, repurposing the model and infrastructure can potentially facilitate a mass vaccination strategy that provides many advantages over the traditional clinic approach in the setting of an ongoing infection in an urban environment.

\section{Operational Differences Between Drive-Through Centers and the Traditional Clinic Model}

The drive-through testing model has been successfully adopted in many countries. Prior to implementation, the model was studied heavily in the context of other disasters. ${ }^{5,6}$ Nevertheless, the reliance on drive-through COVID-19 testing centers has helped scale the ability to test a patient every 3 to 10 minutes $-30 \%$ faster than the traditional clinic method. ${ }^{1,2}$

One of the main advantages of drive-through testing centers is increased efficiency while maintaining the same level of safety to healthcare workers. ${ }^{7}$ In the event of a mass COVID-19 vaccination campaign, there is a high probability that front-line healthcare workers will continue having to deal with the ongoing infectious nature of the virus. This would require the continued use of personal 
protective equipment (PPE). Whereas PPE will have to be replaced much more frequently in a traditional clinical setting, a drive-through center minimizes exposure to a potentially sick patient, which in turn decreases resource use and waste. This claim is supported by findings from the Mayo Clinic, whose drive-through testing center reported a $96 \%$ reduction in mask use, a $97 \%$ reduction in gown use, and a $47 \%$ reduction in glove use. ${ }^{8}$ Given these considerations, the drive-through model is more efficient than the traditional clinic in terms of the number of patients that can be treated per unit of time and the total use of PPE.

\section{Drive-Through Centers Help Overcome Barriers to Mass Vaccinations}

Transitioning drive-through centers from providing COVID-19 testing to include vaccination efforts can help address barriers to vaccine adoption and distribution.

\section{Vaccine Adoption}

Improving vaccine adoption rates is a highly complex, context-specific issue. ${ }^{9}$ Common rationales for avoiding vaccination include decreased trust in authorities and inconvenient access to the vaccine. ${ }^{10}$ Repurposing current drive-through testing center infrastructure takes advantage of the patient's familiarity with the process to improve their confidence in their ability to secure a vaccine efficiently, thus improving its adoption.

\section{Vaccine Distribution}

Consolidating the lessons learned from and building upon the infrastructure of current drive-through testing centers into a decision support system can help improve efficiency upon roll-out of a mass vaccination campaign and provide the opportunity to preemptively address barriers to vaccine distribution. ${ }^{11}$ Evidence from previous vaccination campaigns highlights the need to proactively address equity among racial, ethnic, and socioeconomic groups, as well as enforcing distribution priorities to high-risk groups, like the homeless, elderly and pregnant women. ${ }^{10}$

Seemingly small shortcomings in access can lead to significant gaps in vaccine distribution consequently negatively impacting public health outcomes. ${ }^{4}$ It is worth emphasizing that many drive-through centers were designed primarily to increase testing through operational efficiency. ${ }^{7}$ This approach prioritized finding large parking lots with easy egress over evaluating whether all underserved communities would have equal access to such centers. Thus, future iterations of the drive-through center approach needs to consider optimizing not only operational throughput but also equitable access.

The drive-through center strategy can be refined by considering alternative locations with better public transportation infrastructure, hiring shuttles or leveraging rideshare services to facilitate access to underserved neighborhoods and patient populations. ${ }^{5}$ However, these solutions also introduce complications due to the infectious nature of the disease. With advanced planning, alternative approaches can be devised to overcome such hurdles.

\section{Unique Opportunities Associated with Drive-Through Testing and Vaccination Centers}

While the drive-through center method is regarded as an effective solution in an urban setting, depending on the distribution of the outbreak, it can also be effective in less populated and rural communities. ${ }^{12}$ Consider a scenario wherein a COVID-19 vaccine has been made available. A rapid response mobile unit could deploy a drive-through testing and vaccination center in areas with an unexpected rise of new infections. This would increase local testing capabilities allowing for better contact tracing and increase vaccination rates to help protect the immediate community.

It is important to point out that administering vaccines through drive-through testing centers introduces logistical challenges, especially in situations where it may take longer to vaccinate a child compared to an adult or where a country's health system may require a patient to visit a healthcare professional prior vaccination or may have limited access to one. ${ }^{13,14}$ Hence, adding these layers to the streamlined testing center process could introduce inefficiencies.

That being said, telemedicine has been increasingly adopted throughout this pandemic. Telemedicine enables a "forward triage" strategy, whereby patients can "visit" a physician in a virtual setting before they are scheduled to enter a drive-through center to receive a vaccination. ${ }^{15-17}$ This approach was used in Israel to increase testing throughput and can be adapted for vaccinations. Ultimately, taking advantage of current infrastructure and improving upon current workflows are key to maximizing the benefits and reducing potential bottlenecks associated with a drive-through testing and vaccination center strategy. 


\section{Conclusion}

As the COVID-19 pandemic continues to unfold, the focus on public health interventions expands to include vaccine development. The time required to test, approve and produce a vaccine provides valuable lead-time to analyze and refine distribution strategies. Drive-through testing centers have proven operationally superior to traditional clinic models for expanding testing capacity in a short period of time. With advanced planning, they could evolve into include vaccination efforts that leverage existing infrastructure, reduce resource use, improve efficiency, and address barriers to care to better respond to the pandemic.

\section{Disclosure}

The author has no potential conflicts of interest to disclose.

\section{References}

1. Kim E. Drawing on Israel's experience organizing volunteers to operationalize drive-through coronavirus testing centers. Disaster Med Public Health Prep. 2020;16:1-3. doi:10.1017/dmp.2020.104

2. Kwon KT, Ko J-H, Shin H, Sung M, Kim JY. Drive-through screening center for COVID-19: a safe and efficient screening system agains massive community outbreak. J Korean Med Sci. 2020;35(11):e123. doi:10.3346/jkms.2020.35.e123

3. Walensky RP, Del Rio C. From Mitigation to Containment of the COVID-19 Pandemic: putting the SARS-CoV-2 Genie Back in the Bottle. JAMA. 2020;17. doi:10.1001/jama.2020.6572

4. Plotkin SA. Mass Vaccination: Global Aspects, Progress and Obstacles. Springer; 2006.

5. Baccam P, Willauer D, Krometis J, Ma Y, Sen A, Boechler M. Mass Prophylaxis Dispensing Concerns: traffic and Public Access to PODs. Biosecurity Bioterrorism. 2011;9(2):14.

6. Zerwekh T, McKnight J, Hupert N, Wattson D, Hendrickson L, Lane D. Mass Medication Modeling in Response to Public Health Emergencies: outcomes of a Drive-thru Exercise. J Public Health Manage Practice. 2007;13(1):1. doi:10.1097/00124784-20070100000003
7. Weiss EA, Ngo J, Gilbert GH, Quinn JV. Drive-through medicine: a novel proposal for rapid evaluation of patients during an influenza pandemic. Ann Emerg Med. 2010;55(3):268-273. doi:10.1016/j. annemergmed.2009.11.025

8. Ton AN, Jethwa T, Waters K, Speicher LL, Francis D. COVID-19 drive through testing: an effective strategy for conserving personal protective equipment. Am J Infect Control. 2020; S019665532030208X. doi:10.1016/j.ajic.2020.04.010

9. Osterholm MT, Kelley NS, Sommer A, Belongia EA. Efficacy and effectiveness of influenza vaccines: a systematic review and meta-analysis. Lancet Infect Dis. 2012;12(1):36-44. doi:10.1016/ S1473-3099(11)70295-X

10. Schmid P, Rauber D, Betsch C, Lidolt G, Denker M-L. Barriers of Influenza vaccination intention and behavior - a systematic review of influenza vaccine hesitancy, 2005 - 2016. Cowling BJ, ed. PLoS One. 2017;12(1):e0170550. doi:10.1371/journal.pone.0170550

11. Ramirez-Nafarrate A, Lyon JD, Fowler JW, Araz OM. Point-ofDispensing Location and Capacity Optimization via a Decision Support System. Prod Oper Manag. 2015;24(8):1311-1328. doi:10.1111/poms. 12323

12. Molling PE, Holst TT, Anderson BG, et al. Drive-through satellite testing: an efficient precautionary method of screening patients for SARS-CoV-2 in a rural healthcare setting. Community Health (Bristol). 2020;6.

13. Lee EK, Smalley HK, Zhang Y, Pietz F, Benecke B. Facility location and multi-modality mass dispensing strategies and emergency response for biodefence and infectious disease outbreaks. IJRAM. 2009;12(2/3/4):311. doi:10.1504/IJRAM.2009.025925

14. Banks LL, Crandall C, Esquibel L. Throughput times for adults and children during two drive-through influenza vaccination clinics. Disaster Med Public Health Prep. 2013;7(2):175-181. doi:10.1017/ dmp. 2013.3

15. Duffy S, Lee TH. In-person health care as option B. $N$ Engl J Med. 2018;378(2):104-106. doi:10.1056/NEJMp1710735

16. Lurie N, Carr BG. The role of telehealth in the medical response to disasters. JAMA Intern Med. 2018;178(6):745. doi:10.1001/ jamainternmed.2018.1314

17. Araz OM, Ramirez-Nafarrate A, Jehn M, Wilson FA. The importance of widespread testing for COVID-19 pandemic: systems thinking for drive-through testing sites. Health Sys. 2020;9(2):119-123. doi:10.1080/20476965.2020.1758000
Journal of Multidisciplinary Healthcare

\section{Publish your work in this journal}

The Journal of Multidisciplinary Healthcare is an international, peerreviewed open-access journal that aims to represent and publish research in healthcare areas delivered by practitioners of different disciplines. This includes studies and reviews conducted by multidisciplinary teams as well as research which evaluates the results or conduct of such teams or healthcare processes in general. The journal covers a very wide range of areas and welcomes submissions from practitioners at all levels, from all over the world. The manuscript management system is completely online and includes a very quick and fair peer-review system. Visit http://www.dovepress.com/testimonials. php to read real quotes from published authors. 